c) Dic NOs, die beim Schmelzen des $\mathrm{Cr}^{2} \mathrm{O}^{3}$ mit KO$\times \mathrm{NO}^{3}$ leicht entsteht, slörte mich anfangs sehr; sie oxydirt das $\mathrm{FeO}$ und dessen ungeachtet reducirt sie in saurer Lösung sogleich die $\mathrm{Cr}^{3}$. Endlich gelang es mir, dieselbe durch Kochen der alkalischen Lösung mit Salmiak bis zur neutralen Reaction zu entfernen. Es bildet sich dann $\mathrm{N} \mathrm{H}^{4} \mathrm{O}+\mathrm{NO}^{3}$, das sich in neutralen Flïssigkeiten bei $50^{\circ} \mathrm{C}$. schon in $\mathrm{N}$ und $\mathrm{HO}$ zerlegt.

d) Mangansäure wird ebenfalls durch Kochen der alkalischen Lösung mit Salıniak zerstört. Es scheidet sich $\mathrm{Mn} \mathrm{O}^{2}$ ab, das man abfiltrirt.

e) $\mathrm{FeO}^{3}$, wenn sie sich bilden sollte, wird schon beim Uebergielsen mit heilsem Wasser zerselzt.

Die Methode wurde an saurem chromsaurcm Kali, neulralem chromsaurem Kali, chromsaurem Baryt, chromsaurem Quecksilberoxydul, chromsaurem Chromchlorid, Chromoxyl, Chromalaun geprüft und hierbei sehr gule Resultate erhalten. Ferner wurde ein Chromeisenstein, der 36,2 - 36,4 pC. Chronoxyd gab und Chromocker von Halle der 1,9-2,1 pC. Chromoxyd gab, auf gleiche Weise analysirt.

\title{
Untersuchungen über die Amylreihe;
}

von Heinrich Medlock.

(Gelesen vor der Chemical Society in London.)

Seil die Unlersuchungen von Dumas*) und Cahours**) die chenische Natur des Kartoffelfuselöls und scine Beziehung zu verschiedenen andern Körpern feslgeslellt hahen, isl dieser Körper von vielen Chemilsern studirt worden. Balard *\%ँt),

*) Annalen der Chemie Bd. XIII, S. 80.

*) Ebendaselbst Bd. XXX, S. 288. Bd. XXXVII, S. 164.

***) Ebendaselbst Bd. LII, S. 311. 
Kurtzsch*), H. Kopp**), Kolbe **t) und Rieckher + ), sowie mehrere Andere, sind nach einander mit der Untersuchung des Amylalkohols beschäftigt gewesen; ihren Versuchen verdanken wir die Kenntnils einer beträchllichen Anzahl seiner Verbindungen. Nichtsdestoweniger bietet die Amylreihe noch keineswegs die Vollständigkeit, welche die Methylreihe und besonders die Aethylreihe erreicht hat und es war in der Absicht, verschiedene der noch vorhandenen Lücken auszufüllen, dals Professor Hofmann mich aufforderle, cinige Abkömmlinge des Anylalkohols in seinem Laboratorium zu untersuchen.

Die ursprüngliche Quelle, welche Dumas und Cahours den Stoff ihrer Untersuchungen lieferte, war die alkoholische Gährung der Kartoffeln und daher der Name Kartoffelfuselül. Die späteren Untersuchungen über den Amylalkolıol scheinen sämmtlich mit einem ähnlichen Producte angestellt worden zu seyn, mit Ausnahme von B alard's Versuchen, aus dessen Abhandlung erhellt, dafs der fragliche Stoff glcichfalls in den geringen Weinbrantweinsorten enthalten ist; in der That wird, nachdem dic alkoholischen Producte bereits übergegangen sind, die Destillation der Weintrebern' noch einige Zeit fortgeselzt, so geht ein Oel über, welches nach Balard's Versuchen seiner Hauptmasse nach aus Oenanthsäureäther und Amylalkohol besteht.

Die Substanz, mit welcher ich meine Präparate darstellte, stammte ans der grofsen Destillerie des Herrn Bowerbank in London, woselbst sic sich in grofser Menge aus den letzten Portionen ausscheidet, welche bei der Destillation des gewöhnlichen Kọnbrantweins übergehen. Ich crwähnc dieser That-

*) Erdma nns Journal Bd. XXXI, S. 1.

* *) Diese Anualen Bd, LV, S. 166.

***) Ebendaselbst Bd. LXV, S. 288.

f) Jahrbuch der Pharmacie Bd. XIV, S. 1. 
sache, weil frühere Untersuchungen von aus ähnlichen Quellen erzeugtem Fuselöl (Getreidefuselöl) die Gegenwart von Amylalkohol nicht zu erkennen gegeben hatten. Weder Mulder*) noch Kolbe **) erwähnen in ihren Arbeiten über Getreidefuselöl die Gegenwart dieser Verbindung; es scheint überhaupt, dafs die Versuche dieser beiden sich auf ein anderes, von dem meinigen ganz verschiedenes Product beziehen. Die neben Oeuanthsäure und Oenanthsäure - Aether (Mulder) und neben Margarinsäure (Kolbe) vorhandene ölige Flüssigkeit, welche unter dem Namen Kornöl beschrieben ist, hat in ihrer Zusammensetzung (Mulder) nichts mit dem eigentlichen Fuselöl gemein; wahrscheinlich haben Mulder sowohl als Kolbe ihre Versuche mit dem allerletzten Product angestellt, welches erst gesammelt wurde, nachdem aller Amylalkohol übergegangen war.

Aus den Versuchen, welche ich der Gesellschaft vorzulegen in Begriff bin, geht hervor, dafs in der Brantweindestillation aus Gerste ein Oel erhalten wird, welches vollkommen identisch mit dem Karloffelfuselöl ist. Dieses $0 \mathrm{el}$, wie es aus der Fabrik bezogen wurde, enthielt noch Alkohol und Wasser, aber keine anderen Beimischungen. Bei der Rectification begann es bei $85^{\circ}$ C. zu sieden; nachdem elwa ein Drittheil uibergegangen, war der Siedepunkt auf 132 (Siedepunkt des reinen Amylalkohols) gestiegen, bei welcher Temperatur die übrigen zwei Drittheile deslillirten. Das in Rede stehende Fuselöl kann keine Körper von höherem Siedepunkte enthalten und es blieb daher nur noch übrig, die Zusammensetzung des Destillates zu untersuchen, welches unter $132^{\circ}$ übergegangen war.

Die Chemiker sind in den letzten Jahren mit einer Reihe von Fällen bekannt geworden, in welchen die Bildung von Metacetonsäure und Buttersäure in Folge von Gührungsprocessen

\footnotetext{
*) Diese Annalen Bd. XXIV, S. 248.

*) Ebendaselbst Bd. XLI, S. 35.
} 
beobachlet wurde, die viel mit der Essiggährung gemein haben, obwohl diese Processe in ihren verschiedenen Stadien noch bei weitem nicht mit der Genauigkeit erforscht sind, als der Uebergang des Weingeisles in Essigsäure. Der bemerkenswerthe Parallelismus, welchen Ameisensäure, Essigsäure, Metacetonsäure, Buttersäure und Valeriansäure nach allen Richtungen hin zu erkennen gegeben haben, machen es nicht unwahrscheinlich, dafs der Ergänzung aller dieser Säuren, so of sie wenigstens in Folge von Gährungsprocessen entstehen, die Bildung eines correspondirenden Alkohols vorausgeht, wie wir diefs bei der Ameisensäure, der Essigsäure und Valeriansâure beobachten. Es schien durchaus nicht unmöglich, dafs das Getreidefuselöl neben Weingeist und Amylalkohol noch einen andern Alkohol, vielleicht den der Metacetonsäure oder Buttersãure enthalte. Um diese Frage, auf welche Dr. Hofman n meine Aufmerksamkeit vorzugsweise gelenkt batte, zu entscheiden, rectificirte ich die ersten Destillate mit besonderer Sorgfalt, nachdem ich das Wasser mittelst Chlorcalcium entfernt hatte. Das Oel wurde so zu fünf Graden fraclionirt und der im Anfange übergehende Alkohol, sowie das zuletzt destillirende Fuselöl bei jeder neuen Destillation weggelassen. Auf diese Weise gelang es, das ganze Destillat in Alkohol und Fuselöl zu zerlegen; das von mir untersuchte rohe Fuselöl besteht also nur aus Weingeist, Wasser und Amylalkohol.

Einwirkung des Phosgengases auf Am yloxydhydrat.

\section{Kohlensaures Amyloxyd.}

Das chlorkohlensaure Aethyloxyd, welches von Dumas * ) durch die Einwirkung von Chlorkohlensäure (Phosgengas) auf absoluten Alkohol erhalten wurde, sowie das Urethan (carbo-

*) Annales de Chim. et de Phys. T. LIV. p. 225. 
minsaures Aethyloxyd), welches aus der Behandlung des neuen Aethers mit Ammoniak hervorgeht, sind in der Amylreihe bis jetzt unvertreten. Die Darstellung dieser Amylverbindungen nahm meine Aufnerksąmkeit zuerst in Anspruch.

Fuselöl absorbirt Phosgengas mit grolser Begierde und unter beträchtlicher Wärmeentwicklung. Ein Grm. bedurfte zu seiner Sättigung beiläufig ein Liter Gas, wobei indefs bemerkt werden muls, dafs das Gas nicht absolut frei von atmosphärischer Luft war. Nach vollständiger Sätligung entwickelte sich eine reichliche Menge von Chlorwasserstoffsäure und die gelbe Flüssigkeit scheidet sich in zwei Schichten, von welchen die untere eine kleine Quantität zufälligen Wassers enthält, welches mit Salzsäure gesättigt ist, während die neue Verbindung oben auf schwimmt. Zur Entfernung der aufgelösten Chlorwasserstoffsäure wurde sie mil dem gleichen Volum Wasser geschüttelt, welches nach der Abscheidung mit der Pipetle entfernt ward. Auf diese Weise ward der gröfste Theil der Salzsäure, aber keineswegs die letzlen Spuren entfernt. Ich liefs defshalb die ölige Flüssigkeit noch eine Zeillang über Bleioxyd stehen und trockncle sie endlich mittelst Chlorcalciun. Als die Flüssigkeit nunmehr zur weiteren Reinigung und zur Bestimmung des Siedepunctes von Neuem destillirt wurde, ergab sich sogleich, dafs sie nicht ohne Zersetzung erhitzt werten könnte; sie schwärzte sich unter stürmischer Entwicklung von Kohlensäure und Chlorwasserstoffsäure, während sich der Siedepunct rasch von 150 auf $224^{\circ}$ crhob, wo er stationär wurde.

Um den Schlüssel zu dieser eigenlhüınlichen Zerselzung zı erhallen, ward das gegen das Ende der Operation übergegangene Destillat genauer untersucht. Dieses Product stellte nach nochmaliger Reclification eine klare, durchsichtige Flüssigkeit dar von angenchmem Geruch, sehr verschieden von dem ursprünglichen Geruch, welchen das Fuselöl nach seiner Sälligung mit Phosgengas besafs. Sein specifisches Gewicht war 
0,9144; sein constanter Siedepunct characterisirt es als chemisches Individuum.

Bei der Verbrennung mit Kupferoxyd wurden folgende Zahlen erhalten :

I. 0,2480 Grm. Subslanz gaben 0,5925 Grm. Kohlensäure und 0,2458 Grm. Wasser.

II. 0,2158 Grm. Substanz gaben 0,5162 Grm. Kohlensäure und $0,2110 \mathrm{Grm}$. Wasser.

In Procenten :

I. II.

$\begin{array}{lrr}\text { Kohlenstoff } & 65,15 & 65,23 \\ \text { Wasserstoff } & 11,01 & 10,87 .\end{array}$

Der cinfachsle atomistische Ausdruck dieser Zahlen ist die Formel :

$$
\begin{array}{lll}
\mathrm{C}_{11} & \mathrm{H}_{11} & \mathrm{O}_{3},
\end{array}
$$

wie sich aus folgender Vergleichung der theorelischen Werthe dieser Formel mit dem Mittel der Versuchszahlen ergiebt :

\begin{tabular}{|c|c|c|c|c|c|}
\hline \multirow[b]{2}{*}{11} & \multirow[b]{2}{*}{ Aeq. } & \multirow[b]{2}{*}{ Kohlensloff } & \multicolumn{2}{|c|}{ Theorie } & Mittel d. V \\
\hline & & & 66,00 & 65,34 & 65,19 \\
\hline 11 & $\Rightarrow$ & Wasserstoff & 11,00 & 10,89 & 10,94 \\
\hline 3 & $\eta$ & Sauerstoff & 24,00 & 23,77 & \\
\hline
\end{tabular}

Die neue Verbindung ist demnach kohlensaures Amyloxyd *):

$$
\mathrm{C}_{10} \mathrm{H}_{11} \mathrm{O}, \mathrm{CO}_{2} \text {. }
$$

*) Die Entstehung des kohlensauren Amyloxyds in der von Herrn MedIock beschriebenen Reaction schien eine einfache Methode anzudeuten, die kohlensauren Aether im Allgemeinen zu erhalten. Die Chemiker kennen den bemerkenswerthen, aber zur Zeit noch etwas geheimnifsvollen Procefs, mittelst dessen das kohlensaure Aethyloxyd zuerst von Dr. Ettling dargestellt wurde (diese Annalen Bd. XIX. S. 17), nämlich durch die Einwirkung des Kaliums auf Oxalsäure-Aether. Dic entsprechende Methylverbindung ist bis jetzt noch nicht erforscht worden. Um zu erfahren, ob das chlorkohlensaure Aethyloxyd und Methyloxyd sich bei der Destillation mit Wasser der Amylverbindung 
Hälle die Analyse einen Zweifel über die Natur desselben gelassen, so hätte ihr Verhalten gegen die Alkalien denselben entfernen müssen. Beim Zusammenbringen mit einer alkoholischen Kalilösung entwickelte sich sogleich der erstickende Geruch des Fuselöls, während die Flüssigkeit zu einer Krystallmasse von koblensaurein Kali erstarrt.

Das Verhalten des Phosgengases gegen Fuselöl ist der Einwirkung dieses Gases auf Alkohol vollkommen analog. Es bilden sich Chlorwasserstoflsäure und chlorkohlensaures Amyloxyd.

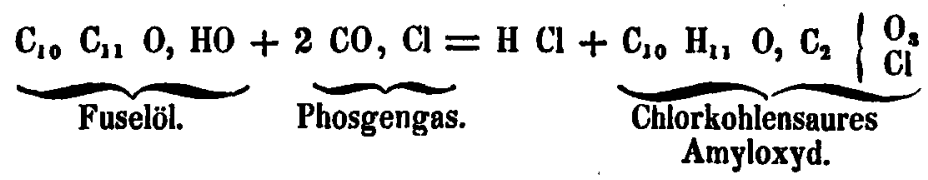

Diese Verbindung ist aber von sehr unstabiler Natur, bei der Beruihrung mit Wasser zerfällt sie sogleich in Chlorwassersloffsäure, Kohlensäure und kohlensaures Amyloxyd. $\mathrm{C}_{10} \mathrm{H}_{\mathrm{J1}} \mathrm{O}, \mathrm{C}_{2}\left\{\begin{array}{l}\mathrm{O}_{3} \\ \mathrm{Cl}\end{array}+\mathrm{HO}=\mathrm{C}_{20} \mathrm{H}_{11} \mathrm{O}, \mathrm{CO}_{2}+\mathrm{HCl}+\mathrm{CO}_{2}\right.$ Chlorkohlensäure-Aether. Kohlensäure-Aether.

Die Verwandlung des Chlorkohlensäure-Aethers war offenbar vorzugsweise während des Waschens mit Wasser vor sich gegangen. Bei einer zweiten Darstellung wurde daher das Waschen ganz unterlassen - nichtsdestoweniger zeigten sich

analog verhalten, veranlafste ich Herrn Ka rl Bl oxam, die Einwirkung des Wassers auf diese Verbindungen zu studiren. Diese Versuche zeigten, dafs der Chlorkohlensăure-Aether in der That eine ähnliche Zersetzung erleidet, dafs aber die Zersetzung viel zu langsam von Statten geht, um eine hequeme Darstellungsmethode abzugeben. Nach fünf bis sechs Destillationen war stets noch eine beträchuliche Menge Chlorkohlensäure-Aethers unzerlegt. Die Gegenwart einer Base, selbst in geringer Quantität, zerstört die Verbindung vollkommen unter Bildung von Chlorid, Carbonat und Alkohol. Es bleibt noch zu versuchen, ob sich das kohlensaure Chlor-Aethyloxyd nicht leichter zersetzt, wenn man es mit Wasser von höherer Temperatur (unter Druck) behandelt.

A. W. Hofmann. 
bei der Destillation der miltelst Bleioxyl von Chlorwasserstoffsäure befreiten Flüssigkeit, dieselben Erscheinungen. Augenscheinlich war die zufällige Feuchtigkeit, welche sich nicht ganz vermeiden lälst, sowie das in der Verwandlung des Bleioxyds in Chlorblei erzeugte Wasser hinreichend gewesen, um die Zersetzung zu bewerkstelligen. Demungeachtet ist es wahrscheinlich, dafs das chlorkohlensaure Amyloxyd existirt und sich bei sorgfältigster Ausschliefsung jeder Art von Feuchtigkeit *) darstellen lassen wird. Der durchdringende Geruch des mit Chlorkohlenoxyd gesättigten Fuselöls, welcher mit dem erstickenden Geruche des letzteren nichts gemein hat, wohl aber dem des Chlorkohlensäure-Aethers gleicht, scheint für diese Ansicht zu sprechen, noch mehr aber das Verhalten des rohen Productes gegen Ammoniak. Man weils, dufs bei der Behandlung von chlorkohlensaurem Aethyloxyd mit Ammoniak der von Dum as mit dem Namen Urethan bezeichnete Körper entsteht, welchen wir jetzt als carbaminsaures Aethyloxyd zu betrachten gewohnt sind. Wird das rohe Product der Einwirkung des Phosgengases auf Fuselöl mit einer alkoholischen Ammoniaklösung vermischt, so bildet sich ein weilser Niederschlag, welcher aus Chlorammonium und einem fettig anzufühlenden, Wallrath ähnlichen Körper besteht. Durch Waschen mit Wasser wird der erstere entfernt, während der letztere im Zustand der Reinheit zurrickbleibt. Leider, obgleich mit ziem-

*) Das Chlor-sowohl als das Kohlenoxyd, welches zur Darstellung des Phosgengases gedient hatte, waren sorgfältig mittelst Schwefelshurure getrocknet worden; auch die mit eingeschliffenen Glasstöpseln versehenen Flaschen waren vollkommen trocken und das Fuselöl war absolut wasserfrei; nichtsdestoweniger schied sich das Oel bei der Săttigung in zwei Schichten, von denen die untere eine wasaserige Chlorwasserstoffsãurelösung war. Es ist klar, dafs dieses Wasser beim Uebergiefsen des zu săttigenden Fuselōls aus einer Flasche in die andere angezogen worden seyn mufs, und da diese Operation bei einer Darstellung 25-30mal wiederholt werden mufste, so läfst sich diefs ohne Schwierigkeit begreifen. 
lich beträchtlicher Menge arbeilend, erhielt ich nicht genug von dieser Substanz zu einer Verbrennung, aber ihr Verhạlten sowohl, als die Analogie erlauben die Annahme, dafs sie das Urethan der Amylreihe war, entstanden durch die Einwirkung des Ammoniaks auf eine kleine Menge unzersetzten chlorkohlensauren Amyloxyds. Gegen diese Annahme lärst sich der Einwurf machen, dals sich das Urethan, wie neuere Versuche von Cahours gezeigt haben, ebenfalls durch die Einwirkung des.Ammoniaks auf kohlensaures Aethyloxyd erhalten läfst. In ersterem Falle aber konnte das kohlensaure Amyloxyd nicht zur Bildung des weilsen Körpers Veranlassung gegeben haben, denn · ich habe diese Verbindung vergeblich mit wässeriger sowohl als alkoholischer Ammoniaklösung in Berührung gelassen, es erfolgte, selbst nach Wochen, keine Einwirkung.

Die Existenz des chlorkohlensauren Amyloxyds ist indessen durch die angeführten Versuche keineswegs festgestellt und ich gedenke dieselben wieder aufzunehmen, sobald das Wetter die Darslellung grofser Mengen von Phosgengas gestallet.

\section{Schwefelcyanamyl.}

Das Interesse, welches die Schwefelcyanverbindungen des Aethyls und Methyls darbieten, ist neuerdings durch die von Dr. Muspratt *) beobachtete Verwandlung derselben in Aethylund Melhylunterschwefelsäure gesteigert worden.

In Folgendem gebe ich meine Versuche über die entsprechenden Amylverbindungen, welche schon im verflossenen Sommersemester angestelit worden, lange ehe ich durch eine Notiz im Journal de Pharmacie erfuhr, dals Herr Hen r y in Frankreich eine Arbeit über denselben Gegenstand unternommen halle.

*) Diese Annalen Bd. LXV. S. 251. 
Die Darstellung des Schwefelcyanamyls ist der der entsprechenden Methyl - und Aelhylverbindungen analog. Eine innige Mischung von zwei Theilen amylschwefelsauren Kalks und einem Theile Schwefelcyankaliums wurden in einer Retorte von dem doppelten Rauminhalte der Destillation unterworfen *). Beim Erwärmen destillirt eine kleine Menge Wassers von höchst eigenthïmlichem Geruche und bei gesteigerter Temperatur geht eine beträchlliche Quantität eines gelben Oeles über, welches denselben Geruch bcsitzt. Dieses Oel ist Schwefelcyanamyl. Zur Reinigung wird es mit Wasser destillirt, alsdann mit der Pipelte abgehoben und mittelst Chlorcalcium getrocknet. Es hält die letzlen Spuren von Wasser mit grofser Hartnäckigkeil zurück das wasserfreie Oel enthält eine grofse Menge Chlorcalcium in Auflösung, von dem es durch Destillation befreit werden mufs.

Das wasserfreie Schwefelcyanamyl begann bei den ersten Destillationen bei $150^{\circ}$ zu sieden, der Siedepunct stieg aber bald auf $195^{\circ}$, bei welcher Temperatur der gröfsere Theil der Substanz überging. Bei der Rectification dieses letzteren Productes wurde ein constanter Siedepunct bei $197^{\circ}$ erhalten. Die folgenden Analysen wurden mit einem bei dieser Temperalur siedenden Oele dargestellt.

I. 0,2816 Grm. Schwefelcyanamyl, mit Kupferoxyd und vorgelegtem Bleioxyd verbrannt, gaben 0,5748 Grm. Kohlensäure und $0,2280 \mathrm{Grm}$. Wasser.

II. 0,2894 Grm. Schwefelcyananyl gaben 0,5914 Grm. Kohlensäure und $0,2320 \mathrm{Grm}$. Wasser.

III. $0,3195 \mathrm{Grm}$. Schwefelcyanamyl, mit Natronkalk verbrannt, gaben 0,5525 Grm. Ammoniumplatinchlorid.

In Procenten :

*) Um starkes Aulschwcllen zu vermeiden, wende man die Salze möglichst trocken an. 
I. II. III.

Kohlenstoff $55,66 \quad 55,73 \quad$ ᄁ

Wasserstoff $8,99 \quad 8,90 \quad$ ᄁ

Slickstoff $\quad$ $\quad 10,84$.

Diese Zahlen führen zu der Formel :

$$
\mathrm{C}_{12} \mathrm{H}_{11} \mathrm{~N} \mathrm{~S}_{2}=\mathrm{C}_{10} \mathrm{H}_{11} \mathrm{Cy} \mathrm{S}_{2} \text {, }
$$

wie sich aus folgender Zusammenstellung ergiebt :

12 Aeq. Kohlenstoff

11 , Wasserstoff

$1 》$ Slickstoff

2 ) Schwefel

1 N Schwe felcyanamyl 129 100,00.

Mittel der Versuche

Theorie

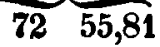

118,52

$14 \quad 10,85$

$14,10,85$
55,69

8,95

10,84

Das reine Schwefelcyananyl ist eine ölige Flüssigkeit von lichtgelber Farbe, welche beim Aufbewahren sich dunkel färbt. Es mischt sich in allen Verhältnissen mit Alkohol und Aether; durch Wasser, worin es beinahe unlöslich ist, wird es aus der Alkohol - und Aetherlösung wieder abgeschieden. Der Siedepunct dieses Körpers liegt genau bei $197^{\circ}$, welche Temperątur ziemlich mit derjenigen übereinstimmt, welche sich aus dem von Cahours *) beobachteten Siedepuncte des Schwefelcyanäthyls berechnet.

$$
146^{\circ}+3 \cdot 19=203
$$

Einwirkung der Salpetersäure auf Schwefelcyanam y 1 .

Gleiche Theile Schwefelcyanamyl und mälsig concentrirte Salpetersäure wurden gemischt und nachdem die erste stürmische Reaction vorüber war, bei einer gelinden Wärme destillirt. Um die Reaction zu vollenden, hat man von Zeit zu Zeit etwas Salpetersäure hinzuzufügen und das Destillat in die Retorte zuriick

\footnotetext{
*) Diese Annalen Bd. LXI. S. 91.
} 
zu gielsen, bis die letzlen Spuren von Schwefelcyanamyl verschwunden sind. Im Verlaufe der Destillation entwickeln sich reichliclıe Mengen Kohlensäure, Stickstoff, Stickstoffoxyd und salpetrige Säure und die Flïssigkeit enthält Schwefelsäure, deren Quantität jedoch mit der Stärke der angewandten Salpetersäure sich ändert; mälsig concentrirte Salpetersäure erzeugt nur eine geringe Menge Schwefelsäure. - Dampft man die Flïssigkeit, nachdem die Reaclion vorüber ist, auf dem Wasserbade ab, so bleibt nach Vertreibung der Salpetersäurc eine rolhe Flüssigkeit von knoblauchartigem Ger'uch und brennendem Geschmack zuriick. Diese Flüssigkeit löst sich im Wasser mit Leichtigkeit; wenn man dic wässerige Lösung nochmals auf dem Wasserbade eindampft, so wird hierdurch die letzte Spur von Salpetersäure enlfernt und dic zurickbleibende Substanz ist beinahe farblos.

Um diese Säure im Zustand der Reinheit zu erhalten, stellte ich das Blcisalz dar, dưch Sälligung der wässerigen Lösung mit kohlensaurem Bleioxyd. Eine Lösung des krystallisirten Bleisalzes mit Schwefelwasserstoff behandelt und nach der Trennung des Schwefelbleis auf dem Wasserbade verdampft, hinterläIst die Säure als eine klare farblose Flüssigkeit, welche in leeren Raume übcr Schwefelsäure allmählig zu einer körnigen Kryslallmasse erstarrt, dic aber, in Berïhrung mit der Luft, sehr rasch Feuchtigkeit anzieht und sich verflüssigt.

Amylunterschwefelsäure, denn diefs ist die in Rede stehende Säure, läfst sich nicht ohne Zersetzung erwärmen. Sie verkohlt unter Entwicklung eines sehr widrigen Geruches, lhre Formel, wie sie sich aus der Analyse verschiedener Salze ergiebt, ist :

$$
\begin{array}{lllll}
H, & \mathrm{C}_{10} & \mathrm{H}_{11} & \mathrm{~S}_{2} & \mathrm{O}_{6}
\end{array}
$$

und geslattet eine Einsicht in die Unbildungsweise des Schwefelcyanamyls. Das Schwefelcyan zerlegt sich, indem der Schwefel durch Salpetersäure oxydirt wird, während das Cyan in der Form von Kohlensäure und Slickstoff entweicht. 


$$
\underbrace{\mathrm{C}_{12} \mathrm{H}_{11} \mathrm{NS}_{2}}+\mathrm{HO}+\mathrm{O}_{0}=\underbrace{\mathrm{H} \mathrm{C}_{10} \mathrm{H}_{12} \mathrm{~S}_{2} \mathrm{O}_{6}}+\mathrm{N}+2 \mathrm{CO}_{2}
$$

Schwefelcyanamyl.

Amylunterscliwefelsäurc.

Der Amylunterschwefelsäure correspondirt die Acthyl- und Melhylunterschwefelsäure ${ }^{*}$ ), welche ror Kurzein von Dr. Musprat $t$ in analogen Reaclionen erhalten wurden; die letztere war hekannllich schon früher von Dr. Kolbe **) als ein Umwandlungsproduct der Chlorkohlenunlerschwefelsäure unter dem Einflusse des galvanischen Stromes aufgefunden worden.

Melhylunterschwefelsïure $\quad \mathrm{H}, \mathrm{C}_{2} \quad \mathrm{H}_{3} \quad \mathrm{~S}_{2} \mathrm{O}_{6}$ $\begin{array}{llllll}\text { Aelhylunterschwefelsäure } \quad \mathrm{H}, \mathrm{C}_{4} & \mathrm{H}_{5} & \mathrm{~S}_{2} & \mathrm{O}_{6}\end{array}$ Amylunterschwefelsäure $\quad \mathrm{H}, \mathrm{C}_{10} \mathrm{H}_{1,} \mathrm{~S}_{2} \mathrm{O}_{0}$.

Ich habe die Verbindung der Amylunterschwefelsäure mit Barium, Kupfer und Blei untersucht.

\section{Amylunterschwefelsaurer Baryt.}

Dieses Salz wird durch Sälligung der wässerigen Säurelösung mit hohlensaurem Baryt crhalten. Die auf dem Wasserbade vcrdampfle Flüssigkeit liefert Krystalle, die sowohl im Wasser als auch im Alkohol aufserordentlich löslich sind. Besser ausgebildele Krystalle können nur durch freiwilliges Verdampfen der wässerigen Lösung erhalten werden. Es schielsen alsdann perlenglänzende Schuppen an.

I. 0,4880 Grm. Salz, bei $100^{\circ}$ getrocknet, gaben 0,4915 Grm. Kohlensïure $=27,46 \mathrm{pC}$. Kohlenstoff und 0,2295 Grm. Wasser $=5,22 \mathrm{pC}$. Wasserstoff.

II. 0,3015 Grm. Sulstanz gaben 0,1599 Grm. schwefelsauren Baryl $=31,11 \mathrm{pC}$. Barium.

Diese Zahlen fïhren zu der Formel :

$$
\mathrm{Ba}, \mathrm{C}_{10} \mathrm{H}_{1 \mathrm{r}} \mathrm{S}_{2} \mathrm{O}_{0} \text {, }
$$

wie sich aus folgender Zusammenslellung ergiebt :

*) Dese Annalen Bd. LXV. S. 251.

**) Ebendaselbst Bd. LlV. S. 174. 
10 Aeq. Kollensloff

11 "Wasserstoff

$2 》$ Schwefel

(j) Sauerstoff

1 " Barium
Theorie

$\overbrace{60,00 \quad 27,31}$

$11,00 \quad 5,00$

$32,00 \quad 14,56$

$48,00 \quad 21,88$

$68,64 \quad 31,25$

Versuche

27,46

5,22

s)

$n$

31,11

1 amylunterschwefels. Baryt 219,64 100,00.

Das Salz ist wasserfrei; ich habe den Wassergehalt bestimmt, welcher bei $100^{\circ}$ weggeht; er ist viel geringer, als pinem Aequivalent entsprechen wiirde *).

\section{Amylunterschoefelsaures Kupferoxyd.}

Dieses Salz läfst sich nur unil Schwierigkeit in Krystallen crhalten. Man bereitet eine Lösung des Salzes durch Sälligung der Sãure mit kohlensaurem Kupferoxyd, verdampft auf dem Wasscrbade zur 'Trockne und zieht den Rückstand mit absolutem

*) Die von IIerrn Medlock dargestellte Săure ist offenbar mit der von Gerathewohl und Erdinann (Erdmann's Journal Bd. XXXIV. S. 447) durch die Einwirkung der Salpetersăure auf das Amylmercaptan erhaltenen Säure identisch, olggleich diese beiden Chemiker eine verschiedene Formel für ihre Verbindung aufstellten. Die für das Barytsalz gegebene Formel :

$\mathrm{Ba}_{\mathrm{a}}, \mathrm{C}_{10} \mathrm{II}_{11} \mathrm{~S}_{2} \mathrm{O}_{3}, \mathrm{IIO}=\mathrm{Ba}, \mathrm{C}_{10} \mathrm{IH}_{12} \mathrm{~S}_{2} \mathrm{O}_{\mathrm{f}}$

unterscheidet sich von Herrn Medlock's Formel :

$$
\mathrm{Ba}, \mathrm{C}_{10} \mathrm{II}_{21} \mathrm{~S}_{2} \mathrm{O}_{6}
$$

nur durch 1 Aeq. Wasscrstoff. Allein in vier Wasserstoffbestimmungen stellte sich ein Verlust heraus, welcher zivischen $0,2 \mathrm{pC}$. mul 0,4 pC. schwankte, wahhrend doch last alle Analysen einen kleinen Ueberschurs geben. Ein ähnlicher Einwand lärst sich gegen die Analyse versehiedener Salze der durch Einwirkung von Salpetersäure auf das Aethylmercaptan dargestellten Säure erheben. Es wăre von Interesse, die Analyse der Barytrerbindung zu wiederholen, auf welche sich die für diese Säure angenomnene Formel :

$$
\mathrm{H}, \mathrm{C}_{4} \mathrm{H}_{5} \mathrm{~S}_{2} \mathbf{O}_{3}
$$

stützt. Es ist sehr wahrscheinlich, dafs sich die Ansicht des Herrn Gerhard hinsichtlich dieser Säure bestätigen wird.

\section{A. W. Hofmann.}


Alkohol aus. Die filtrirle Lösung liefert bei freiwilliger Verdunstung in engen Gefâlsen krystallinische Blättchen.

I. 0,4960 Grm. Salz, mit chromsaurem Bleioxyd verbrannt, gaben $0,5936 \mathrm{Grm}$. Kohlensâure $=32,63 \mathrm{pC}$. Kohlenstoff und $0,2754 \mathrm{Grm}$. Wasser $=6,16 \mathrm{pC}$. Wasserstoff.

II. 0,3970 Grm. Substanz linterliefsen 0,0863 Grm. Kupferoxyd $=17,33 \mathrm{pC}$. Kupfer.

Diese Zahlen führen zu der Formel :

$$
\mathrm{Cu}, \mathrm{C}_{10} \mathrm{H}_{11} \mathrm{~S}_{2} \mathrm{O}_{6} \text {, }
$$

wie folgende Berechnung zeigt :

10 Aeq. Kohlenstoff

$11 》$ Wasserstoff

2 " Schwefel

$6 \cap$ Sauerstoff

$1 \rightarrow$ Kupler

1 amylunterschwefels. Kupfer $182,66100,00$.

\begin{tabular}{rrc} 
Theorie & Vers. \\
\hline 60,00 & 32,84 & 32,64 \\
11,00 & 6,02 & 6,16 \\
32,00 & 17,52 & $n$ \\
48,00 & 26,29 & n \\
31,66 & 17,33 & 17,33
\end{tabular}

Dieses Salz enthält kein Krystallwasser.

\section{Amylunterschwefelsaures Bleioxyd.}

Es wird wie die beiden vorhergehenden Salze dargestellt. Die wässerige Lösung schiefst bei freiwilligem Verdampfen in seidigen Nadeln an, welche aufserordenllich löslich sind. Beim Erhitzen entwickeln diese Krystalle einen höchst widerlichen Geruch, während Schwefelblei zurückbleibt.

I. 0,5690 Grm. Salz, mit chromsaurem Bleioxyd verbrannt, gaben $0,4936 \mathrm{Grm}$. Kohlensäure $=23,65 \mathrm{pC}$. Kohlenstoff und 0,2168 Grm. Wasser $=4,23$ pC. Wasserstoff.

II. 0,3340 Grm. Substanz gaben 0,1986 Grm. Schwefelsäureblei $=40,63$ pC. Blei.

Diese Versuche entsprechen der Formel :

$$
\mathrm{Pb}, \mathrm{C}_{10} \mathrm{H}_{\mathrm{II}} \mathrm{S}_{2} \mathrm{O}_{\mathrm{a}} \text {. }
$$




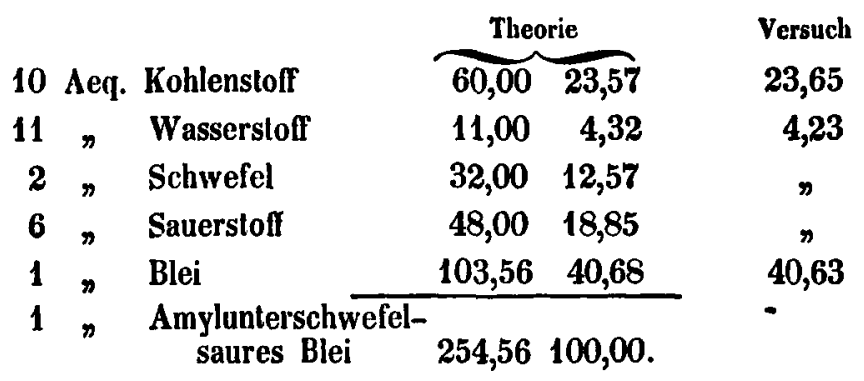

Dieses Salz ist ebenfalls wasserfrei.

Zum Schlusse folgende Uebersicht der von mir untersuchten Verbindungen der Amylunterschwefelsäure :

$\begin{array}{llllll}\text { Barytsalz } & \mathrm{Ba}, & \mathrm{C}_{10} & \mathrm{H}_{11} & \mathrm{~S}_{2} & \mathrm{O}_{0} \\ \text { Kupfcrsalz } & \mathrm{Cn}, & \mathrm{C}_{10} & \mathrm{H}_{11} & \mathrm{~S}_{2} & \mathrm{O}_{6} \\ \text { Bleisalz } & \mathrm{Pb}, \mathrm{C}_{10} & \mathrm{H}_{11} & \mathrm{~S}_{2} & \mathrm{O}_{6} .\end{array}$

Ich bereitete noch verschiedene Glieder der Amylreihe, enthalte mich aber aller Delails, da deren Analyse noch nicht vollendet ist. Nur diels sey noch bemerkt, dafs ich das Verhalten des Kaliums gegen Cyanamyl und die Einwirkung des Chlorzinks auf Fuselöl studirt habe. In dem ersteren Processe bildet sich neben gasförmigen Producten eine schöne organische Base, welche dem Kyanethin correspondirl; in dem lelzteren habe ich aufser der flüssigen Kohlenwasserstoffsäure des Herrn Balard cinen gasförmigen Kohlenwasserstoff von der Zusammensetzung des ölbildenden Gases erhalten, welcher frühér nicht beobachlet wurde.

Eine ausführliche Beschreibung dieser Verbindungen werde ich der Gesellschaft demnächst vorlegen.

Ich schliefse diese Mittheilung mit dem Ausdruck des lebhaftesten Dankes, den ich Herrn Professor Hofmann schulde für seinen Rath und seine Hülfe bei Anstellung der beschriebenen Versuche. 